\title{
Nuclear Reactions That Occur in Human Body During Proton Therapy
}

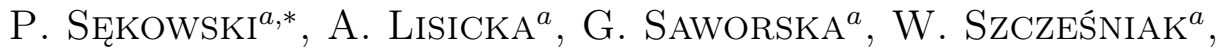 \\ T. Matulewicz ${ }^{a}$, I. Skwira-Chalot ${ }^{a}$, A. Spyra ${ }^{a}$, \\ T. ChORWACIK ${ }^{b}$, J. SWAKOŃ ${ }^{b}$ AND J.W. Mietelski ${ }^{b}$ \\ ${ }^{a}$ Faculty of Physics, University of Warsaw, \\ L. Pasteura 5, 02-093 Warsaw, Poland \\ ${ }^{b}$ Institute of Nuclear Physics, Polish Academy of Sciences, \\ W.E. Radzikowskiego 152, 31-342 Kraków, Poland
}

Doi: 10.12693/APhysPolA.139.454 *e-mail: przemyslaw.sekowski@fuw.edu.pl

\begin{abstract}
The depth dose profile of the proton beam used in tumour treatment saves healthy tissues. Together with high biological effectiveness, proton therapy is a very promising type of tumour radiotherapy. Contrary to $\mathrm{X} / \gamma$ beams, protons may easily induce nuclear reactions leaving radioactive isotopes after irradiation. Measurement of the secondary particles along with the determination of the time during which those nuclei remain in the tissues allows to estimate their impact on the therapeutic effects. Animal tissues (heart, liver, and bone) were irradiated with $60 \mathrm{MeV}$ proton beam with different doses at the AIC-144 cyclotron of the Institute of Nuclear Physics PAS. The produced radionuclides were measured via their decay, allowing to estimate the radioactivity of the studied tissues.
\end{abstract}

topics: induced radioactivity, proton therapy, secondary radiation

\section{Introduction}

The therapy using a proton (carbon) beam is advantageous as compared to the photon irradiation of tumour cells, as it maximises the deposited dose at the end of the range in tissue (the Bragg peak). The intensity of the proton beam in tissue is reduced only by nuclear reactions until protons are finally stopped around the nominal range. The nuclear reactions on nuclei forming the tissue may create radioactive nuclei that quickly undergo $\beta^{+}$ decay. This decay generates positrons, which annihilate with electrons and emit (in most cases) two photons. These photons can be used in positron emission tomography (PET) carried out simultaneously with hadron therapy to control the position of the deposited radiation [1]. Nuclear reactions induced by protons used for the therapy have not been studied yet in all details. Also, the model calculations, based on the Monte Carlo simulations, are not fully consistent (see Fig. 6 in [2]). Produced radioactive nuclei can decay and thus deposit an additional dose in tissue.

\section{Proton-induced radiation}

The human (animal) tissue is composed mainly of compounds of atoms containing light nuclei [3]. Apart from the dominant hydrogen, only three elements are above $1 \%$ of the total number of atoms:
TABLE I

Selected nuclear reactions on light nuclei abundant in human tissue.

\begin{tabular}{|c|c|c|c|}
\hline Reaction & $\begin{array}{c}Q \text {-value } \\
{[\mathrm{MeV}]}\end{array}$ & Residue & $T_{1 / 2}[\mathrm{~min}]$ \\
\hline${ }^{12} \mathrm{C}(p, d)$ & -16.5 & \multirow{3}{*}{${ }^{11} \mathrm{C}$} & \multirow{3}{*}{$20.36(2)$} \\
\hline${ }^{12} \mathrm{C}(p, p n)$ & -18.7 & & \\
\hline${ }^{14} \mathrm{~N}(p, \alpha)$ & -2.9 & & \\
\hline${ }^{14} \mathrm{~N}(p, d)$ & -8.3 & \multirow{3}{*}{${ }^{13} \mathrm{~N}$} & \multirow{3}{*}{$9.965(4)$} \\
\hline${ }^{14} \mathrm{~N}(p, p n)$ & -10.6 & & \\
\hline${ }^{16} \mathrm{O}(p, \alpha)$ & -5.2 & & \\
\hline${ }^{16} \mathrm{O}(p, d)$ & -13.4 & \multirow[t]{2}{*}{${ }^{15} \mathrm{O}$} & \multirow{2}{*}{$2.041(6)$} \\
\hline${ }^{16} \mathrm{O}(p, p n)$ & -15.7 & & \\
\hline
\end{tabular}

oxygen, carbon, and nitrogen. Calcium and phosphorus are at the $0.2 \%$ level, while all other elements contribute in total below $0.5 \%$. The proton reactions on light nuclei may lead to the creation of a few radioisotopes, that decay by the $\beta^{+}$process within a few minutes. The most important ones, with production cross-sections in the range of tens $\mathrm{mb}$, are listed in Table I.

Note that the $\beta^{+}$decay of nuclei listed as products in Table I leads directly to the ground state of stable isotopes [4]. The emission of $e^{+}$dominates the decay mode, as the electron capture process is 
at the level of $10^{-3}$. In this case, the $e^{+} e^{-}$annihilation process is the evident procedure to observe the production of those nuclei. The $e^{+}$stopping process and the eventual photoelectric or Compton scattering of annihilation quanta contribute to the dose deposited in irradiated tissue, apart from the dominant effect of slowing down the energetic proton.

\subsection{Gamma rays}

As the decay of all reaction products mentioned in Table I leads to the $e^{+} e^{-}$annihilation and consequently to the emission of $511 \mathrm{keV}$ quanta (principal channel), the only distinction of the created activities might be obtained through the deconvolution of the decay curve. Obviously, individual determination of cross-sections of numerous reactions listed partially in Table I is not feasible, when the "target" is as complex as tissue.

The decay of these reaction products can be measured just after the end of irradiation. In order to study the position (energy) dependence, the irradiated tissue was composed of several slices (irradiated simultaneously). The energy range of protons in individual slices (see Fig. 1) was obtained with the use of the Ziegler formula [5]. After irradiation, the samples were placed on a rotating wheel of the detection setup equipped with up to six scintillation counters [6]. Rotation of the wheel allowed that the samples were measured in the identical detection system (but not at the same time). This procedure allows for obtaining the decay curve for all samples simultaneously irradiated.

The experimental decay of $511 \mathrm{keV}$ activity of a slice of pig heart tissue (see Fig. 2) for measurements shorter than $1000 \mathrm{~s}$ can be described as individual contributions from decays of three nuclides: ${ }^{11} \mathrm{C},{ }^{13} \mathrm{~N}$, and ${ }^{15} \mathrm{O}$. For longer measurements, the oxygen contribution is negligible and can be omitted.

As the activity of the neighbouring samples significantly influences the measurement of the one placed between the detectors [6], the detectors were equipped with lead shields to partially reduce this unwanted effect. These shields reduce the effect

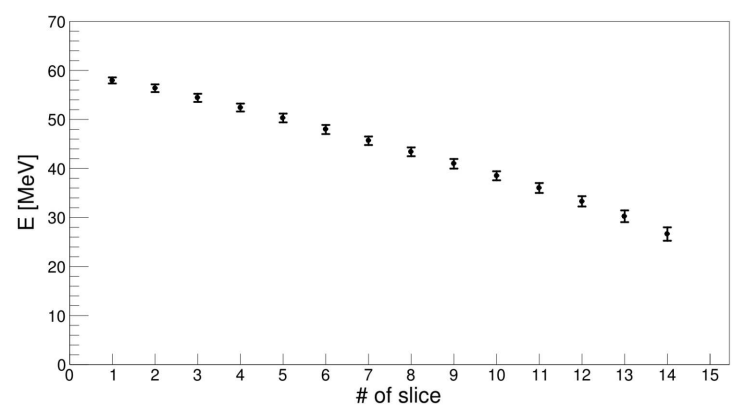

Fig. 1. Mean energy range of protons in individual slices of pig heart tissue (example of one target set).

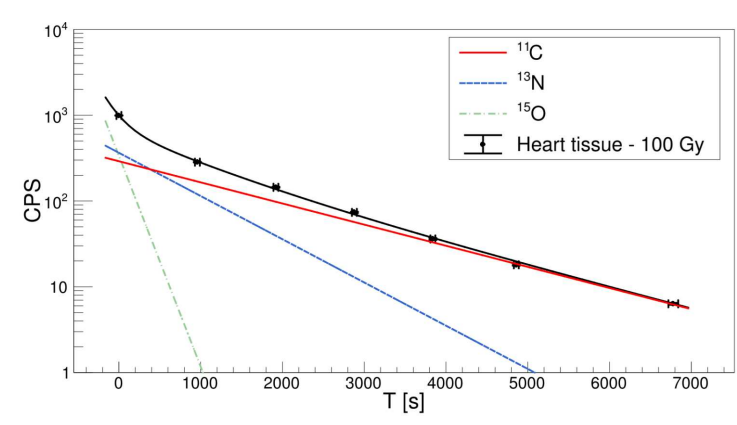

Fig. 2. Decay of $511 \mathrm{keV}$ activity of a slice of heart tissue, measured with a $\mathrm{LaBr}_{3}$ detector. Individual contributions from decays of ${ }^{11} \mathrm{C},{ }^{13} \mathrm{~N}$, and ${ }^{15} \mathrm{O}$ are indicated. The fitted curve (black solid line) assumes the decay constants as in Table I.

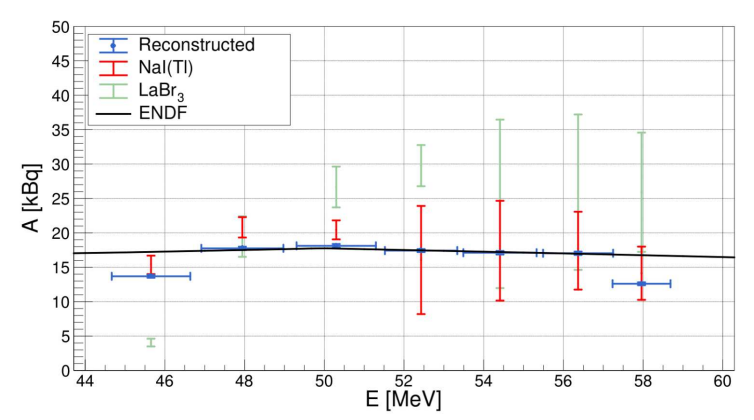

Fig. 3. The $511 \mathrm{keV}$ activity of ${ }^{11} \mathrm{C}$ of slices of pig heart tissue at the end of bombardment with $60 \mathrm{MeV}$ protons providing a $100 \mathrm{~Gy}$ dose. The range of activities determined from individual $\mathrm{NaI}(\mathrm{Tl})$ and $\mathrm{LaBr}_{3}$ detectors are indicated. The solid line denotes activity obtained from cross-section values based on ENDF base, tissue composition, and beam parameters.

mentioned, but the results still have to be corrected (work in progress). Subsequently, the comparison of the results with the expected yields might confirm that no other $\beta^{+}$activities are produced.

The short-lived activities $\left({ }^{13} \mathrm{~N}\right.$ and $\left.{ }^{15} \mathrm{O}\right)$ can be registered only during the initial phase of the measurement (very few points on the decay curve) and the extracted activity suffers from large uncertainty. This is not the case of ${ }^{11} \mathrm{C}$ activity, which can be extrapolated to the time of the end of bombardment. The ${ }^{11} \mathrm{C}$ activities of several slices of a pig heart (with an average mass of $0.6 \mathrm{~g}$ ), shown in Fig. 3, have been obtained from the analysis of spectra from $\mathrm{LaBr}_{3}$ and $\mathrm{NaI}(\mathrm{Tl})$ detectors. The decomposition of the decay curve to three components was not always unambiguous, so two options have been studied: $\left({ }^{11} \mathrm{C}\right.$ and $\left.{ }^{13} \mathrm{~N}\right)$ or $\left({ }^{11} \mathrm{C}\right.$ and $\left.{ }^{15} \mathrm{O}\right)$. It leads to the range of activities indicated in Fig. 3. Another approach takes into account the influence of neighbouring samples, determining the activity of the sample through the nonnegative least squares method with configurationspecific efficiency correction. 
As it can be seen in Fig. 3, both approaches are in good agreement with the ENDF evaluation (accounting for target composition [3] and beam specifications). The results from the $\mathrm{LaBr}_{3}$ detector, which does not have any collimator, are apparently higher as compared to the results from the $\mathrm{NaI}(\mathrm{Tl})$ detector, which was equipped with a lead collimator reducing the influence of the neighbouring samples. The activities reconstructed by the non-negative least squares method with neighbour correction are more precise than the results obtained from the standard decay fitting.

The long-time activities were studied by placing the irradiated sample on a HPGe detector in a lowbackground environment. The measurements [7] showed that isotopes like ${ }^{34 m} \mathrm{Cl}$ or ${ }^{44} \mathrm{Sc}$ were produced during irradiation of bone.

\subsection{Neutrons}

While the radiation emitted after the $\beta^{+}$decay of the reaction product is localised within the range of $1 \mathrm{~cm}$ [7], the neutrons might penetrate a longer distance. The presence of neutrons was indicated in the following measurement. A sample of a pig liver placed in a metal tube (aluminium, copper, or steel) was irradiated with a proton beam so that only the tissue was exposed. Neutron-induced activities of ${ }^{56} \mathrm{Mn}$ or ${ }^{61} \mathrm{Cu}$ in metal tubes were detected in a HPGe spectrometer localised in a low-background environment. The registered activity was approximately twice the activity measured in the reference experiment, where only the tube without tissue was exposed, so the proton beam passed freely.

These results motivate us to measure the energy spectrum of neutrons emitted from tissues during irradiation. For that new measurement, the setup based on eight liquid scintillation detectors BC501A will be used. The energy calibration of the setup will follow the technique described in [8]. Currently, the knowledge of the neutron flux and energy in the irradiation zone of AIC-144 cyclotron is based on the Monte-Carlo simulation [9]. The neutron spectral fluences were established to be site-dependent [10] in experiments detecting thermal neutrons. Experiments with the new setup will attempt to evaluate the neutron yield from the irradiated sample.

\section{Conclusions}

Animal tissues (heart, liver, and bone) were irradiated with a $60 \mathrm{MeV}$ proton beam with different doses at the AIC-144 cyclotron of the Institute of Nuclear Physics PAS. The radionuclides ${ }^{11} \mathrm{C},{ }^{13} \mathrm{~N}$ and ${ }^{15} \mathrm{O}\left(T_{1 / 2}\right.$ in the range of $\left.2-20 \mathrm{~min}\right)$ were detected via their $\beta^{+}$decay in a setup allowing to measure many slices of the tissue simultaneously irradiated. The activity of slices of a pig heart determined with $\mathrm{LaBr}_{3}$ and $\mathrm{NaI}(\mathrm{Tl})$ scintillators agrees with the predictions based on ENDF cross-section values. Longer-lived activities were observed employing HPGe detectors placed in a low-background environment.

Initial measurements using neutron-activation indicate the presence of neutrons resulting from nuclear reactions in tissue. Further measurements using dedicated neutron spectrometers are planned.

\section{References}

[1] M. Durante, H. Paganetti, Rep. Prog. Phys. 79, 096702 (2016.

[2] P. Sękowski, I. Skwira-Chalot, A. Spyra, T. Matulewicz, Acta Phys. Pol. B 51, 873 (2020).

[3] H. Woodard, D. White, Brit. J. Radiol. 59, 708 (1986).

[4] G. Audi, F.G. Kondev, M. Wang, B. Pfeiffer, X. Sun, J. Blachot, M. MacCormick, Chin. Phys. C 36, 1157 (2012).

[5] J.F. Ziegler, J.P. Biersack, Nucl. Instrum. Methods Phys. Res. B 268, 1818 (2010).

[6] T. Matulewicz, P. Sękowski, I. SkwiraChalot, J. Tarasiuk, Acta Phys. Pol. B 51, 1939 (2020).

[7] P. Sękowski, I. Skwira-Chalot, T. Matulewicz, Acta Phys. Pol. B 49, 681 (2018).

[8] J. Yan, R. Liu, C. Li, L. Jiang, X.X. Lu, T.H. Zhou, Chin. Phys. C 34, 993 (2010).

[9] T. Cywicka-Jakiel, L. Stolarczyk, J. Swakon,, P. Olko, M.P.R. Waligórski, Radiat. Meas. 45, 1127 (2010).

[10] X. Yan, U. Titt, A.M. Koehler, W.D. Newhauser, Nucl. Instrum. Methods Phys. Res. A 476, 429 (2002). 